\title{
Concord grape juice supplementation improves memory function in older adults with mild cognitive impairment
}

\author{
Robert Krikorian $^{1}$, Tiffany A. Nash ${ }^{1}$, Marcelle D. Shidler ${ }^{1}$, Barbara Shukitt-Hale ${ }^{2}$ and James A. Joseph ${ }^{2}$ \\ ${ }^{1}$ Department of Psychiatry, University of Cincinnati Academic Health Center, Cincinnati, OH, USA \\ ${ }^{2}$ USDA Human Nutrition Research Center on Aging and Tufts University, Boston, MA, USA
}

(Received 1 May 2009 - Revised 3 September 2009 - Accepted 4 September 2009 - First published online 23 December 2009)

Concord grape juice contains polyphenol compounds, which have antioxidant and anti-inflammatory properties and influence neuronal signalling. Concord grape juice supplementation has been shown to reduce inflammation, blood pressure and vascular pathology in individuals with CVD, and consumption of such flavonoid-containing foods is associated with a reduced risk for dementia. In addition, preliminary animal data have indicated improvement in memory and motor function with grape juice supplementation, suggesting potential for cognitive benefit in ageing humans. In this initial investigation of neurocognitive effects, we enrolled twelve older adults with memory decline but not dementia in a randomised, placebo-controlled, double-blind trial with Concord grape juice supplementation for 12 weeks. We observed significant improvement in a measure of verbal learning and non-significant enhancement of verbal and spatial recall. There was no appreciable effect of the intervention on depressive symptoms and no effect on weight or waist circumference. A small increase in fasting insulin was observed for those consuming grape juice. These preliminary findings suggest that supplementation with Concord grape juice may enhance cognitive function for older adults with early memory decline and establish a basis for more comprehensive investigations to evaluate potential benefit and assess mechanisms of action.

Memory: Cognitive impairment: Elderly: Grape juice: Polyphenols

Worldwide dementia prevalence is almost 25 million cases and is projected to reach more than 81 million cases by the year $2040^{(1)}$. Alzheimer's disease comprises 60 to $80 \%$ of cases of dementia ${ }^{(2)}$. The construct mild cognitive impairment ${ }^{(3)}$ identifies individuals with elevated risk for dementia $^{(4)}$, and progression from mild cognitive impairment to Alzheimer's disease can be as high as $10 \%$ per year ${ }^{(5)}$. Further, there are indications that even age-associated memory impairment, originally conceptualised as benign forgetfulness ${ }^{(6,7)}$, can reflect very early neurodegeneration. Older adult samples with subjective memory complaints who meet criteria for age-associated memory impairment show degradation in the medial temporal lobe that is similar, albeit not as extensive, as that observed in subjects with mild cognitive impairment and Alzheimer's disease ${ }^{(8)}$, and longitudinal investigation has shown a trebling of risk for those categorised as having age-associated memory impairment ${ }^{(9,10)}$. Such findings imply that memory complaints and associated manifestations in everyday functioning can be meaningful indicators of neurodegeneration. Preventive interventions initiated when early memory decline is evident have the potential to forestall progression, most likely at the final stage when such treatment might be effective ${ }^{(11)}$.

Regulation of inflammation generally is reduced with ageing $^{(12)}$, and accelerated inflammation is implicated in neurodegenerative disorders such as Alzheimer's disease ${ }^{(13)}$. Berry fruits contain polyphenol compounds, which have anti-inflammatory and antioxidant properties ${ }^{(14)}$. Polyphenols also induce neuroprotective effects and influence neuronal signalling involved in memory function ${ }^{(14-16)}$, and specific constituents of grape juice have exhibited neuroprotective effects $^{(17)}$.

Concord grape juice contains a variety of flavonoids and antioxidants, among them anthocyanins and proanthocyanidins $^{(18,19)}$ and comparatively high levels of total phenolics ${ }^{(20)}$. Information concerning flavonoid transport into the central nervous system and absorption into brain tissue is emerging. A number of recent studies have indicated that certain of these compounds, in particular anthocyanins, cross the blood-brain barrier, although specific mechanisms have not been established ${ }^{(21-23)}$. In addition, anthocyanins have been identified in brain regions that mediate cognition, including the medial temporal lobe and cortex ${ }^{(24)}$, and hippocampal distribution has been associated with behavioural enhancement in animal supplementation studies ${ }^{(24,25)}$.

Human trials have shown that short- and moderate-term supplementation with grape juice produces benefit in individuals with CVD, including increased serum antioxidant capacity and reduced LDL oxidation ${ }^{(19)}$, improved endothelial function ${ }^{(26)}$ and reduced platelet aggregation ${ }^{(27)}$. Such findings are pertinent with respect to age-related cognitive decline because of the strong relationship between CVD and neurodegeneration $^{(28-31)}$. Epidemiological studies indicate that consumption of fruits and vegetables is associated with lower risk of neurodegenerative disorders and better cognitive performance in the elderly ${ }^{(32-34)}$, and these effects have been 
attributed to the intake of a variety of flavonoid compounds with antioxidant and anti-inflammatory properties. Recently, a preliminary animal study demonstrated that ingestion of Concord grape juice for 6-8 weeks induced enhancement of cognitive performance in aged rodents ${ }^{(35)}$.

We sought to assess the effect of supplementation with Concord grape juice on memory performance in older adults with early age-related memory decline in a controlled trial as an initial assessment of potential benefit in an at-risk sample. We also obtained data on mood, anthropometrics and metabolic parameters.

\section{Methods \\ Participants}

Participants were recruited from the general community with newspaper advertising soliciting older adults with early memory decline but not dementia for a dietary intervention study. We enrolled twelve participants (eight men, four women) with acquired memory changes such as forgetfulness and prospective memory lapses. The mean age of the entire sample was 78.2 (SD 5.0) years and the mean educational level was $14 \cdot 1$ (SD 2.9) years.

\section{Procedure}

Prospective participants were assessed with structured interview instruments to determine eligibility for study inclusion. The Academic and Medical History Questionnaire ${ }^{(36)}$ was used to obtain demographic information and information regarding academic attainment, current and past medical conditions, and medication and substance use. Those with diabetes, substance-abuse disorder, or diagnosed psychiatric or neurological condition were excluded. The level of memory impairment was determined with the Clinical Dementia Rating ${ }^{(37)}$, which elicits information from the participant and an informant (typically, spouse or adult child) about the nature and extent of cognitive decline as manifested in activities at home and in the community. The domains memory, orientation, problem solving, community affairs, home activities and personal care were evaluated to determine a dementia staging classification. Scores for each domain contributed to a global Clinical Dementia Rating classification with the memory domain weighted most heavily. Clinical Dementia Rating classifications include no impairment, mild decline, and mild, moderate and severe dementia. We enrolled individuals with mild decline and excluded those with Clinical Dementia Rating classifications indicating no impairment and those with mild, moderate and severe dementia. A sum of boxes score also was derived ${ }^{(38)}$. This score represented the arithmetic sum of the category scores across the six domains of functioning and served to quantify level of functional decline.

Seven subjects were randomly assigned to receive the placebo beverage and five were assigned to receive $100 \%$ Concord grape juice. Placebo and juice were provided for the research by Welch Foods, Inc. (Concord, MA, USA). The placebo beverage contained no juice or natural polyphenol but was formulated to look and taste like grape juice and to have the same carbohydrate composition and energy load $(3.0 \mathrm{~kJ} / \mathrm{ml})$. The intervention involved 12 weeks of daily consumption of juice or placebo with assessments at pre-treatment baseline and during the final week of the intervention. Previous human trials examining antioxidant effects, endothelial function and cardioprotection in healthy subjects and those with CVD used briefer interventions, of the order of $2-4$ weeks $^{(17,20-21)}$. We chose a longer intervention period because our outcomes concerned cognitive-cerebral function in older adults, and there are indications in preclinical studies with other berry fruits that several weeks may be required for accumulation in brain regions ${ }^{(39)}$. We instituted a dosing schedule determined by body weight to maintain daily consumption between 6 and $9 \mathrm{ml} / \mathrm{kg}$, a range consistent with other human grape juice trials ${ }^{(17,20-21)}$. Individuals weighing 54 to $64 \mathrm{~kg}$ were prescribed $444 \mathrm{ml} / \mathrm{d}$, those weighing between 65 and $76 \mathrm{~kg}$ consumed $532 \mathrm{ml} / \mathrm{d}$, and those weighing between 77 and $91 \mathrm{~kg}$ consumed $621 \mathrm{ml} / \mathrm{d}$. Participants were instructed to take daily quantities in equal, divided dosages with the morning, midday and evening meals.

The primary outcomes were neurocognitive measures of memory function administered before and after the intervention. The California Verbal Learning Test ${ }^{(40)}$ was administered to assess verbal learning and retention, and the Spatial Paired Associate Learning Test ${ }^{(41)}$ was used to evaluate non-verbal memory. The California Verbal Learning Test is a listlearning and recall task, and the Spatial Paired Associate Learning Test assesses memory for visual-spatial information that is not amenable to verbal encoding. Both list-learning and paired associate tasks have been used in the context of cognitive ageing and dementia and are among the more sensitive measures of memory decline associated with neurodegeneration ${ }^{(42-44)}$. We also assessed mood as a potential covariate of the cognitive measures with the Geriatric Depression $\mathrm{Scale}^{(45)}$. We performed weight and waist circumference measures and obtained fasting blood samples for determination of serum glucose and insulin values.

Analyses of covariance were performed for each outcome factor to isolate effects of the intervention while controlling for individual differences ${ }^{(46)}$. The outcome score from the final visit was the dependent measure and the corresponding score from the baseline visit and the depressive symptom score were covariate measures. We used eta squared values to derive Cohen's $f$ effect size estimates, which are characterised as small $(0 \cdot 10)$, medium $(0 \cdot 25)$ and large $(0 \cdot 40)^{(47)}$.

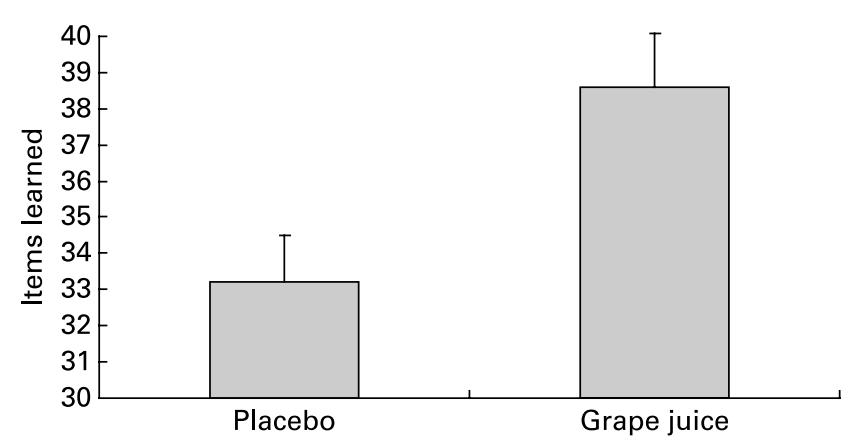

Fig. 1. List acquisition performance assessing verbal learning on the California Verbal Learning Test. Values are adjusted means, with standard errors represented by vertical bars. Subjects consuming Concord grape juice demonstrated significant improvement $(F(1,8)=5.55 ; P=0.04$; Cohen's $f=0 \cdot 28)$. 


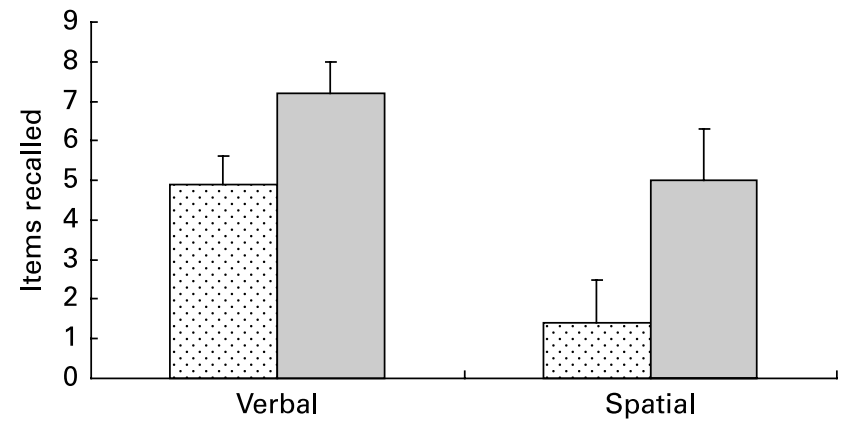

Fig. 2. Delayed recall performance for verbal material on the California Verbal Learning Test $(F(1,8)=3.37 ; P=0.10$; Cohen's $f=0.35)$ and for visual-spatial material on the Spatial Paired Associate task $(F(1,8)=3.23$; $P=0.12$; Cohen's $f=0.67)$. Subjects consumed either Concord grape juice $(\square)$ or a placebo drink (웅). Values are adjusted means, with standard errors represented by vertical bars

The present study was conducted according to the guidelines laid down in the Declaration of Helsinki and all procedures involving human subjects were approved by the University of Cincinnati Medical Institutional Review Board. Written informed consent was obtained from all subjects.

\section{Results}

At pre-intervention baseline there was a modest, nonsignificant difference for age between the groups $(80 v .75$ years; $t(10)=1 \cdot 8 ; P=0 \cdot 10)$. There was no group difference for educational level $(13.4$ v. 15.2 years; $t(10)=1.02$; $P=0 \cdot 32$ ), index of functional impairment (Clinical Dementia Rating sum of boxes score $1.0 v .1 .0 ; t(10)=0.0 ; P=1.0)$, weight $(74.3$ v. $79.4 \mathrm{~kg} ; t(10)=1.04 ; P=0.32)$ and waist circumference $(92.7$ v. $96.7 \mathrm{~cm} ; t(10)=0.81 ; \quad P=0.43)$. There was a group difference for level of depressive symptoms (Geriatric Depression Scale score 7.8 v. 3.0; $t(10)=2 \cdot 19 ; P=0 \cdot 05)$, with greater depressive symptomology among the placebo subjects. However, the symptom level was not clinically elevated for either group ${ }^{(48)}$.

Both the juice and placebo beverage were generally well tolerated, and there was no consistently reported adverse effect. Discrete concerns included, for example, increased frequency of urination associated with greater fluid consumption and aversion to the taste of the juice or placebo that developed over time.

As shown in Fig. 1, analysis of covariance demonstrated a significant effect $(P=0.04)$ for item acquisition across learning trials on the California Verbal Learning Test, indicating improvement for subjects in the Concord grape juice group relative to those receiving placebo. The effect size was moderate (Cohen's $f=0 \cdot 28$ ). Also, there were trends toward improved performances for the grape juice subjects with respect to delayed verbal recall $(P=0.10$; Cohen's $f=0.33)$ and spatial memory $(P=0 \cdot 12$; Cohen's $f=0 \cdot 67)$, although these were not statistically significant (Fig. 2).

There was no appreciable effect of the intervention on depressive symptoms (adjusted Geriatric Depression Scale scores $5.0 v$ v. $7 \cdot 2 ; F(1,8)=2.56 ; P=0.14)$ and no effect on weight $(77.5$ v. $77.8 \mathrm{~kg}$, adjusted values; $F(1,8)=0.31$; $P=0.58)$ or waist circumference $(94.9 v .95 .5 \mathrm{~cm}$, adjusted values; $F(1,8)=0 \cdot 24 ; P=063)$. Fasting glucose values were not affected by the intervention (1011 v. $975 \mathrm{mg} / \mathrm{l}$, adjusted values; $F(1,8)=0.42 ; P=0.53)$, but fasting insulin at 12 weeks was significantly elevated for the subjects consuming grape juice $(10.0$ v. $13.7 \mu \mathrm{U} / \mathrm{ml}$, adjusted values; $F(1,8)=6 \cdot 07 ; P=0 \cdot 03)$. Table 1 contains the unadjusted mean scores for the outcome measures and shows the changes in absolute values from the baseline to final assessment.

\section{Discussion}

In this preliminary study we sought to assess the effect of moderate-term supplementation with $100 \%$ Concord grape juice on cognition in older adults with early memory decline and found that memory function was improved with regular grape juice consumption. To our knowledge, this is the first controlled human trial examining neurocognitive response to this dietary intervention, and our findings are consistent with those of a recent animal study showing improvement in cognitive performance with grape juice supplementation in aged rodents ${ }^{(35)}$. Our data do not provide information as to possible mechanisms leading to the beneficial effects. However, given the existing body of research concerning reductions of inflammatory and oxidative stress markers in human subjects with CVD and lower risk of age-related neurodegeneration with flavonoid consumption, these putative mechanisms would be primary considerations.

Table 1. Unadjusted mean values for memory, mood, anthropometric and metabolic measures by group*

\begin{tabular}{|c|c|c|c|c|c|c|}
\hline & \multicolumn{3}{|c|}{ Placebo ( $n 7)$} & \multicolumn{3}{|c|}{ Concord grape juice $(n 5)$} \\
\hline & Baseline & Final & Difference & Baseline & Final & Difference \\
\hline CVLT learning & $33 \cdot 2$ & $33 \cdot 2$ & 0.0 & $35 \cdot 2$ & $38 \cdot 6$ & 3.4 \\
\hline CVLT recall & $5 \cdot 4$ & $5 \cdot 0$ & -0.4 & $6 \cdot 0$ & $7 \cdot 2$ & $1 \cdot 2$ \\
\hline S-PAL & $2 \cdot 4$ & $2 \cdot 0$ & -0.4 & $2 \cdot 8$ & 4.5 & $1 \cdot 7$ \\
\hline GDS & $7 \cdot 8$ & $7 \cdot 2$ & -0.6 & 3.0 & $5 \cdot 0$ & $2 \cdot 0$ \\
\hline Weight (kg) & $74 \cdot 3$ & 74.9 & 0.6 & $79 \cdot 4$ & $80 \cdot 4$ & 1.0 \\
\hline Waist (cm) & $92 \cdot 7$ & 93.0 & 0.3 & $96 \cdot 7$ & $97 \cdot 5$ & 0.8 \\
\hline Glucose (mg/l) & 1002 & 999 & -3 & 915 & 987 & 72 \\
\hline Insulin $(\mu \mathrm{U} / \mathrm{ml})$ & 11.9 & $11 \cdot 1$ & -0.8 & $9 \cdot 6$ & $12 \cdot 6$ & $3 \cdot 0$ \\
\hline
\end{tabular}

CVLT, California Verbal Learning Test; S-PAL, Spatial Paired Associate Learning Test; GDS, Geriatric Depression Scale.

*Baseline refers to measures obtained at the pre-intervention assessment. Final refers to measures obtained during the final week of the intervention. Difference $=$ final score less baseline score. 
Recent preliminary data involving pharmaceutical TNF- $\alpha$ inhibition have suggested that acute functional improvement can be observed in patients with Alzheimer's disease ${ }^{(49,50)}$, indicating that suppression of a pro-inflammatory cytokine can ameliorate mental decline even in patients with substantially more advanced neurodegeneration than in our sample of mild cognitive impairment subjects. Accordingly, consistent application of a food product with anti-inflammatory effects over a brief to moderate timeframe also might be expected to induce cognitive-cerebral enhancement, especially in individuals with very early neurodegeneration. Pre-clinical data indicating absorption of anthocyanins in brain regions mediating cognition and associations with behavioural indices of cognitive function ${ }^{(24,25)}$ also would support the notion that the demonstrated improvement in memory ability may reflect reduced inflammation and/or enhanced neural function in response to the intervention. While it is not yet clear to what extent and by what mechanism berry fruit constituents cross the blood-brain barrier, anthocyanins have been identified in specific brain tissues even when not detected in plasma ${ }^{(51,52)}$. And, it may be that consistent, moderate-term consumption is necessary to achieve sufficient concentrations in brain sites ${ }^{(39)}$. Further study of Concord grape juice supplementation for greater duration with memory and inflammatory marker outcomes will be important. Other putative mechanisms including reduction of oxidative stress and enhanced neuronal signalling also merit investigation, as these factors have been demonstrated to be important in animal studies with blueberry supplementation ${ }^{(53,54)}$.

Our preliminary data indicated increased fasting insulin for those who received grape juice in the absence of changes in weight and waist circumference. This finding was unanticipated and is provocative given the matched carbohydrate load in the placebo beverage and the fact that group differences in metabolic parameters were not observed before the intervention. It is possible that increased insulin secretion was induced by constituents of the grape juice other than sugars. There are data indicating that anthocyanins derived from blueberries influence metabolic function, in particular enhancing the actions of insulin ${ }^{(54,55)}$. It may be that similar or related actions were induced in the present study, with consistent grape juice consumption resulting in increased insulin secretion. However, such notions are speculative, particularly given the small scale of this preliminary trial. Certainly, this issue warrants further investigation with respect to its reproducibility and the specific nature and basis for the effect.

The major limitation of the present study was the small sample size, which limited power to detect differences. However, the moderate to large effect sizes indicate that it would be worthwhile to conduct larger trials to evaluate the neurocognitive benefits and putative mechanisms of Concord grape juice supplementation in pre-dementia conditions. In view of the public health burden associated with neurodegeneration and Alzheimer's disease, in particular, safe, low-cost dietary interventions offer the possibility of inducing substantial benefit.

\section{Acknowledgements}

Funding and material support was provided by Welch Foods, Inc. (Concord, MA, USA).
R. K. conceived of the study and supervised the data collection, analyses, interpretation and manuscript preparation. T. A. N. and M. D. S. participated in data collection, interpretation and manuscript preparation. B. S.-H. and J. A. J. participated in manuscript preparation.

None of the authors has a financial interest in the supporting company or the outcome of the research activity.

\section{References}

1. Ferri CP, Prince M, Brayne C, et al. (2005) Global prevalence of dementia: a Delphi consensus study. Lancet 366, 2112-2117.

2. Alzheimer's Association (2008) 2008 Alzheimer's disease facts and figures. Alzheimers Dement 4, 110-133.

3. Petersen RC (2004) Mild cognitive impairment as a diagnostic entity. J Intern Med 256, 183-194.

4. Chertkow H, Nasreddine A, Joanette Y, et al. (2007) Mild cognitive impairment and cognitive impairment, no dementia: part A, concepts and diagnosis. Alzheimers Dement 3, 266-282.

5. Mitchell AJ \& Shiri-Feshki M (2009) Rate of progression of mild cognitive impairment to dementia - meta-analysis of 41 robust inception cohort studies. Acta Psychiatr Scand 119, $252-265$.

6. Crook TH, Bartus RT, Ferris SH, et al. (1986) Age-associated memory impairment: proposed diagnostic criteria and measures of clinical change. Dev Neuropsychol 2, 261-276.

7. Neilsen H, Lolk A \& Kragh-Sorensen P (1998) Age-associated memory impairment - pathological memory decline or normal aging? Scand J Psychol 39, 33-37.

8. Goldman WP \& Morris JC (2001) Evidence that age-associated memory impairment is not a normal variant of aging. Alzheimer Dis Assoc Disord 15, 72-79.

9. Reid LM \& MacLullich AMJ (2006) Subjective memory complaints and cognitive impairment in older people. Dement Geriatr Cogn Disord 22, 471-485.

10. Saykin AJ, Wishart HA, Rabin LA, et al. (2006) Older adults with cognitive complaints show brain atrophy similar to that of amnestic MCI. Neurology 76, 834-842.

11. Cotman CW (2000) Homeostatic processes in brain aging: the role of apoptosis, inflammation, and oxidative stress in regulating healthy neural circuitry in the aging brain. In The Aging Mind: Opportunities in Cognitive Research, pp. 114-143 [P Stern and L Carstensen, editors]. Washington, DC: National Academy Press.

12. Franceschi C, Capri M, Monti D, et al. (2007) Inflammaging and anti-inflammaging: a systematic perspective on aging and longevity emerged from studies in humans. Mech Ageing Dev 128, $92-105$.

13. Giunta B, Fernandez F, Nilolic WV, et al. (2008) Inflammaging as a prodrome to Alzheimer's disease. J Neuroinflammation $\mathbf{5}$, $51-65$.

14. Ishige K, Schubert D \& Sagar Y (2001) Flavonoids protect neuronal cells by three different mechanisms. Free Radic Biol Med 30, 433-446.

15. Joseph JA, Arendash G, Gordon M, et al. (2003) Blueberry supplementation enhances signaling and prevents behavioral deficits in an Alzheimer's disease model. Nutr Neurosci 6, $153-162$

16. Olszanecki R, Gebska A, Kozlovski VI, et al. (2002) Flavonoids and nitric oxide synthase. $J$ Physiol Pharm 53, 571-584.

17. Conte A, Pellegrini S \& Tagliazucchi D (2003) Synergistic protection of PC12 cells from $\beta$-amyloid toxicity by resveratrol and catechin. Brain Res Bull 62, 29-38.

18. O'Byrne DJ, Devaraj S, Grundy SM, et al. (2002) Comparison of the antioxidant effects of Concord grape juice flavonoids and 
$\alpha$-tocopherol on markers of oxidative stress in healthy adult. Am J Clin Nutr 76, 1367-1374.

19. Rice-Evans CA, Miller NJ \& Paganga G (1996) Structureantioxidant activity relationships of flavonoids and phenolic acids. Free Radic Biol Med 20, 933-956.

20. Mullin W, Marks SC \& Crozier A (2007) Evaluation of phenolic compounds in commercial fruit juices and fruit drinks. J Agric Food Chem 55, 3148-3157.

21. Singh M, Arseneault M, Sanderson T, et al. (2008) Challenges for research on polyphenols from foods in Alzheimer's disease bioavailability, metabolism, and cellular and molecular mechanisms. J Agric Food Chem 56, 4855-4873.

22. Youdim KA, Shukitt-Hale B \& Joseph JA (2004) Flavonoids and the brain: interactions at the blood-brain barrier and their physiological effects on the central nervous system. Free Radic Biol Med 37, 1683-1693.

23. Youdim KA, Qaiser MZ, Begley D, et al. (2004) Flavonoid permeability across an in situ model of the blood-brain barrier. Free Radic Biol Med 36, 592-604.

24. Andres-Lacueva C, Shukitt-Hale B, Galli RL, et al. (2005) Anthocyanins in aged blueberry-fed rats are found centrally and may enhance memory. Nutr Neurosci 8, 111-120.

25. Williams CM, El Moshen MA, Vauzour D, et al. (2008) Blueberry-induced changes in spatial working memory correlate with changes in hippocampal CREB phosphorylation and brain-derived neurotrophic factor (BDNF) levels. Free Radic Biol Med 45, 295-305.

26. Chou EJ, Keevil JG, Aeschlimann S, et al. (2001) Effect of ingestion of purple grape juice on endothelial function in patients with coronary heart disease. Am $J$ Cardiol 88 , $553-555$

27. Freedman JE, Parker C, Li L, et al. (2001) Select flavonoids and whole juice from purple grapes inhibit platelet function and enhance nitric oxide release. Circulation 103, 2792-2798.

28. Kalaria RN (2000) The role of cerebral ischemia in Alzheimer's disease. Neurobiol Aging 21, 321-330.

29. Rober AE, Garani Z, Alexandrov AV, et al. (2006) Interaction of cardiovascular disease and neurodegeneration: transcranial Doppler ultrasonography and Alzheimer's disease. Neurol Res 28, 672-678.

30. Skoog I \& Gustafson D (2003) Hypertension, hypertensionclustering factors and Alzheimer's disease. Neurol Res $\mathbf{2 5}$, $675-680$

31. Swan FE, Carmelli D \& La Rue A (1998) Systolic blood pressure tracking over 25 to 30 years and cognitive performance in older adults. Stroke 29, 2334-2340.

32. Dai Q, Bornstein AR, Wu Y, et al. (2006) Fruit and vegetable juices and Alzheimer's disease: The Kame Project. Am J Med 119, 751-759.

33. Nurk E, Refsum H, Drevon CA, et al. (2009) Intake of flavonoid-rich wine, tea and chocolate by elderly men and women is associated with better cognitive test performance. J Nutr 139, 120-127.

34. Letenneur L, Proust-Lima C, Le Gouge A, et al. (2007) Flavonoid intake and cognitive decline over a 10 -year period. Am J Epidemiol 165, 1364-1371.

35. Shukitt-Hale B, Carey A, Simon L, et al. (2006) Effects of Concord grape juice on cognitive and motor deficits in aging. Nutrition 22, 295-302.
36. Krikorian R, Zimmerman ME \& Fleck DE (2004) Inhibitory control in obsessive-compulsive disorder. Brain Cogn 54, 257-259.

37. Hughes CP, Berg L, Danziger WL, et al. (1982) A new clinical scale for the staging of dementia. Br J Psychiatry 140, 566-572.

38. Lynch CA, Walsh C, Blanco A, et al. (2006) The clinical dementia rating sum of box score in mild dementia. Dement Geriatr Cogn Disord 21, 40-43.

39. Willis LM, Shukitt-Hale B \& Joseph JA (2009) Recent advances in berry supplementation and age-related cognitive decline. Cur Opin Clin Nutr Metab Care 12, 91-94.

40. Delis DC, Kramer JH, Kaplan E, et al. (2000) California Verbal Learning Test, 2nd ed. San Antonio, TX: Psychological Corp.

41. Krikorian R (1996) Independence of verbal and spatial paired associate learning. Brain Cogn 32, 219-223.

42. Greenaway MC, Lacritz LH, Binegar D, et al. (2006) Patterns of verbal memory performance in mild cognitive impairment, Alzheimer's disease, and normal aging. Cogn Behav Neurol 19, 79-84.

43. Fowler KS, Saling MM, Conway EL, et al. (2001) Paired associate performance in the early detection of DAT. J Int Neuropsychol Soc 8, 58-71.

44. Sahakian B, Downes J, Eagger S, et al. (1990) Sparing of attentional relative to mnemonic function in a subgroup of patients with dementia of the Alzheimer's type. Neuropsychologia 28, 1197-1213.

45. Yesavage JA, Brink TL, Rose TL, et al. (1983) Development and validation of a geriatric depresssion rating scale: a preliminary report. J Psychiatr Res 17, 37-49.

46. Sheeber LB, Sorensen ED \& Howe SR (1996) Data analytic techniques for treatment outcome studies with pretest/posttest measurements: an extensive primer. J Psychiatr Res 30, 185-199.

47. Cohen J (1988) Statistical Power Analysis for the Behavioral Sciences, 2nd ed. New York: Lawrence Erlbaum Associates.

48. Olin JT, Schneider LS, Eaton EM, et al. (1992) The Geriatric Depression Scale and the Beck Depression Inventory as screening instruments in an older adult outpatient population. Psychol Assess 4, 190-192.

49. Griffin WS (2008) Perispinal etanercept: potential as an Alzheimer therapeutic. J Neuroinflammation 5, 3.

50. Tobinick EL \& Gross H (2008) Rapid cognitive improvement in Alzheimer's disease following perispinal etanercept administration. J Neuroinflammation $\mathbf{5}, 2$.

51. Shukitt-Hale B, Lau FC \& Joseph JA (2008) Berry fruit supplementation and the aging brain. J Agric Food Chem 56, 636-641.

52. Kalt W, Blumberg JB, McDonald JE, et al. (2008) Identification of anthocyanins in the liver, eye, and brain of blueberry-fed pigs. J Agric Food Chem 56, 705-712.

53. Casadesus G, Shukitt-Hale B, Stellwagen HM, et al. (2004) Modulation of hippocampal plasticity and cognitive behavior by short-term blueberry supplementation in aged rats. Nutr Neurosci 7, 309-316.

54. Martineau LC, Couture A, Spoor D, et al. (2006) Anti-diabetic properties of the Canadian lowbush blueberry Vaccinium angustifolium Ait. Phytomedicine 13, 612-623.

55. Tsuda $\mathrm{T}$ (2008) Regulation of adipocyte function by anthocyanins: possibility of preventing the metabolic syndrome. J Agric Food Chem 56, 642-646. 